\title{
The Consequences of a Strengthened Newtonian Gravity at Short Distances
}

\author{
Lenser Aghalovyan \\ Institute of Mechanics of National Academy of Sciences of Armenia, Yerevan, Armenia \\ Email: lagal@sci.am
}

How to cite this paper: Aghalovyan, L. (2018) The Consequences of a Strengthened Newtonian Gravity at Short Distances. International Journal of Astronomy and Astrophysics, 8, 191-199. https://doi.org/10.4236/ijaa.2018.82014

Received: April 13, 2018

Accepted: June 18, 2018

Published: June 21, 2018

Copyright $\odot 2018$ by author and Scientific Research Publishing Inc. This work is licensed under the Creative Commons Attribution International License (CC BY 4.0).

http://creativecommons.org/licenses/by/4.0/ (c) (i) Open Access

\begin{abstract}
A central interaction of bodies which at short distances is stronger than Newtonian is considered. It is shown that the escape velocity from a body is essentially more than the escape velocity given by Newton's theory. The connection of the new central interaction with the gravitational radius of a black hole is found. It is shown that the gravitational radius of a black hole may be arbitrarily large.
\end{abstract}

\section{Keywords}

Gravitation, Central Interaction, Potential Field, Black Hole

\section{Introduction}

The construction of the theory of movement of the solar planet system was a triumph of science in the middle ages, especially in Astronomy and Mechanics. The confrontation between the supporters of Ptolemaic geocentric system and heliocentric system of Aristarchus-Copernicus lasting for centuries ended with the victory of the latter. In the third-century B.C. the representative of the Pythagorean School of Greece Aristarchus of Samos has advanced heliocentric system of the planetary motion, but it was rejected by the ancient astronomers as baseless in their opinion. Further, from all the systems of the world, created in antiquity for the explanation of the planetary motion, the most famous one was geocentric system of Ptolemy who lived in Alexandria in the II century A.D. From the observations of the starry sky the ancients concluded that it moves around our Earth, which is motionless and is in the center of the Universe. In Ptolemy system everything is explained with the help of circumferences and circular motions. Yet, this system which had existed for more than thousand years, turned out to be very complicated and often was in contradiction with the data 
of astronomical observations which had become intensive after the invention of a telescope by Galileo Galilei. Eighteen centuries after Aristarchus in XVI century Copernicus revived the heliocentric model and unlike Aristarchus who had only given the general idea developed the details of the heliocentric system and bases of the calculations of planetary positions. Yet, Copernicus went on relying on Ptolemy method of the circular orbits and he failed to build a comprehensive theory. A radical change in the victory of the heliocentric system in the beginning of the XVII century was made by Johann Kepler who, using the wonderful catalogue of the data of the exclusively exact observations about the planets motion, especially the data about Mars, made up by Tycho Brahe, formulated his three famous laws on the motion of the planets. According to Kepler's first law any planet moves around the Sun in an elliptical orbit with the Sun in one of the foci of an ellipse (with this Ptolemy model of motion was neglected). According to the second law any planet moves in the orbit with constant sectorial velocity. Kepler's third law establishes connection between the big semi-axis (a) of the ellipse and the period $(T)$ by which the planet completes a full turnover $\left(T^{2} / a^{3}=4 \pi^{2} /(G(M+m))\right), G$ is the gravitational constant.

Kepler's laws are empiric. Several decades later Newton brought out Kepler's laws and formulated the famous law of Universal Gravitation. According to Newton's law the force of Universal Gravitation is central, each mass $m$ is attracted by another mass $M$ in the Universe with force, inversely proportional to the quadrate of distance between the masses, and is directed along the line, connecting the centers of the masses. Newton did much more, proving that the orbit of the bodies, moving around the Sun may be any of the curves of the conic sections family (circle, ellipse, parabola, hyperbola). In the next decades and centuries Newton's law of Universal Gravitation received a lot of convincing and vivid confirmations. Applying this law William Hershel discovered the planet Uranus in 1781, in 1840-s Adams in England and Le Verrier in France discovered the planet Neptune.

In the XVIII century Halley's Comet and lots of small planet-asteroids were discovered. Yet, in spite of the great success some phenomena were difficult to explain by Newton's law. In 1859 Le Verrier discovered some discrepancy of the orbit of the nearest to the Sun planet Merkuri in the perihelion with the results of the observations. Not finding any convincing explanation of this fact, in 1895 Simon Newcomb expressed an opinion that possibly Newton's law of inverse squares was not performed precisely at small distances. In 1917 the fact connected with the anomaly of the orbit of Merkuri was explained on the base of Einstein's general theory of relativity (GTR) and the contradiction seemed run out. But in 1965 it was proved by R.Dicke and M.Goldenberg that the Sun is not round and its polar diameter is $35 \mathrm{~km}$ less than equatorial one which permitted to explain the residual displacement of Merkuri's perihelion almost by $10 \%$.

It put under hesitation the compliance of GTR with the results of the observations ([1] [2] [3]). The various variants of central forces, under which the solu- 
tion of the motion equation is brought to the quadratures, are discussed by Jakobi, Bertran, Darbu, Alfen [4]. Yet, they haven't described at least one really existing motion [4]. The basic types of really existing nowadays in the nature and Atomic Physics forces are noted in [1] [5]. The second characteristic of the current situation is connected with the question of existence of "Dark Bodies" (in the modern terminology "Black Holes"). In 1783 English amateur astronomer John Mitchell, at a later date one of the founders of Seismology, and in 1795 well-known French Mathematician and Mechanician Laplass, independently of each other on the base of Newton's law of gravitation expressed an opinion that in nature bodies for which the necessary velocity for overcoming their gravitation exceeds the speed of light should exist $(c)$. That is why such bodies should be "dark". Bodies like that are invisible and they can be discovered indirectly by the gravitational influence on other bodies. Mitchell and Laplace derived the radius of "the black body" $r_{g}$ (gravitational radius) at its given mass, using the idea of the second cosmic (escape) velocity $r_{g}=2 G M / c^{2}$. After building GTR Mitchell and Laplass discussions were subjected to the critics in the sense that at close to the speed of light velocities the formulae of classical Mechanics are not applicable, though on both theories one and the same value for the gravitational radius is obtained. And the opponents of GTR claim that it is not applicable, as long as the solution of the equations of this theory contains a singularity unacceptable when discovering natural phenomena.

The facts, brought above, determine the relevance of the question raised by Newcomb, namely: if there is such a central interaction which at small distances differs from Newtonian one (it is stronger), and coincides with it at big distances. Below we shall give a positive answer to the posed question.

\section{On More Powerful Central Interaction of Bodies}

Let us consider a version of central interaction of bodies, which at short distances describes more powerful interaction, comparing with Newtonian gravitational one, and practically coincides with it at comparatively big distances. Let us have bodies with masses $m, M$. Put the beginning of the polar coordinates $(r, \theta)$ in the center of the body with mass $M$. The central force of the interaction will be given in the form of

$$
\boldsymbol{F}=-G m M \frac{\mathrm{e}^{k / r}}{r^{2}} \frac{\boldsymbol{r}}{|\boldsymbol{r}|},
$$

or

$$
F=-G m M \frac{\mathrm{e}^{k / r}}{r^{2}} .
$$

where $G$ is the gravitational constant in Newton's law of gravitation

$\left(G=6.67 \times 10^{-11} \mathrm{~m}^{3} /\left(\mathrm{kg} \cdot \mathrm{s}^{2}\right)\right)$. Index $k$ will characterize the power (intensity) of the gravity center. At $k=0$ the interaction (1) coincides with Newtonian one (coefficient $G m M$ is chosen for it). And if $k>0$, it will be more powerful than 
Newtonian interaction. It is obvious that $k$ has dimensionality of the length. We shall speak about its possible values a little bit later. The interaction (1), in somewhat different notations, was previously considered by us [6].

As long as the processes, taking place in the universe, as a rule, are periodical, we shall below prove the possibility of the periodical solution existence under the interaction as well Formula (1).

The field made by force $\boldsymbol{F}$, given in Formula (1) is potential with potential

$$
U=-\frac{G m M \mathrm{e}^{k / r}}{k}+\text { const },
$$

which is essentially stronger than the potential of Newton field $(U=-G m M / r)$. As force $\boldsymbol{F}$ is central, the trajectory of the material point is plane curve and the law of the squares takes place:

$$
r^{2} \frac{\mathrm{d} \theta}{\mathrm{d} t}=C
$$

where $C$ is equal to the initial velocity moment relatively to the center of gravity. Taking into account (4), the velocity of the trajectory point is determined by formulae

$$
v^{2}=C^{2}\left(\left(\frac{\mathrm{d}(1 / r)}{\mathrm{d} \theta}\right)^{2}+(1 / r)^{2}\right) .
$$

Using the theorem on kinetic energy $\left(\mathrm{d} m v^{2} / 2=F \mathrm{~d} r\right)$, we have

$$
v^{2}=\frac{2 G M}{k} \mathrm{e}^{k / r}+h
$$

where the constant of integration $h$ is determined from the initial condition at $r=r_{0}, v=v_{0}$.

Denote $\psi=1 / r$, then

$$
v^{2}=C^{2}\left(\left(\frac{\mathrm{d} \psi}{\mathrm{d} \theta}\right)^{2}+\psi^{2}\right)
$$

and from (6), (7) it follows

$$
\left(\frac{\mathrm{d} \psi}{\mathrm{d} \theta}\right)^{2}=\left(\frac{2 G M}{k} \mathrm{e}^{k \psi}+h\right) \frac{1}{C^{2}}-\psi^{2} .
$$

The determination of the trajectory in the Polar system $(r, \theta)$ is brought to the calculation of the integral

$$
\int_{\theta_{0}}^{\theta} \mathrm{d} \theta= \pm \int_{\psi_{0}}^{\psi} \frac{\mathrm{d} \psi}{\sqrt{\left(\frac{2 G M}{k} \mathrm{e}^{k \psi}+h\right) \frac{1}{C^{2}}-\psi^{2}}}
$$

For the clarification of the trajectory form decompose function ( $\mathrm{e}^{k \mu}$ ) into Maclaurin series and confine ourselves with the first three summands for now

$$
\mathrm{e}^{k \psi} \approx 1+k \psi+\frac{1}{2} k^{2} \psi^{2}
$$

According to (8) we shall have 


$$
\begin{gathered}
\left(\frac{\mathrm{d} \psi}{\mathrm{d} \theta}\right)^{2}=\delta_{1}\left[k_{2}^{2}-\left(\psi-k_{1}\right)^{2}\right], \\
\delta_{1}=1-\frac{G M k}{C^{2}}, k_{1}=\frac{G M}{C^{2} \delta_{1}} \\
k_{2}^{2}=k_{1}^{2}+(2 G M+k h) \frac{1}{k C^{2} \delta_{1}}=\frac{1}{k C^{2} \delta_{1}^{2}}\left[G M+(G M+k h) \delta_{1}\right]
\end{gathered}
$$

We shall consider $\delta_{1}>0$, for only in this case the solution will be periodical and the trajectory will be conic section. Putting in the designations $\psi-k_{1}=k_{2} \rho$, Equation (11) will have the form of

$$
\left(\frac{\mathrm{d} \rho}{\mathrm{d} \theta}\right)^{2}=\delta_{1}\left(1-\rho^{2}\right)
$$

from where it follows

$$
\rho=\cos \sqrt{\delta_{1}}\left(\theta-\theta_{0}\right)
$$

Returning to the initial designations, we shall have

$$
\begin{aligned}
& \psi=k_{1}+k_{2} \cos \sqrt{\delta_{1}}\left(\theta-\theta_{0}\right), \\
& r=\frac{1 / k_{1}}{1+\left(k_{2} / k_{1}\right) \cos \sqrt{\delta_{1}}\left(\theta-\theta_{0}\right)},
\end{aligned}
$$

i.e., the trajectory is conic section with parameters

$$
\begin{gathered}
p=\frac{1}{k_{1}}=\frac{C^{2}}{G M}-k, \\
\varepsilon=\frac{k_{2}}{k_{1}}=\sqrt{1+\left(\frac{C^{2}}{M G k}-1\right)\left(2+\frac{h k}{M G}\right) .}
\end{gathered}
$$

\section{On Possible Orbits of Bodies under the New Central Interaction (1)}

As $\delta_{1}>0$, then $C^{2} /(M G k)-1>0$. In order to get an elliptic orbit, it is necessary that $2+h k /(M G)<0$, i.e.

$$
h<-2 G M / k \text {. }
$$

The trajectory will be an ellipse if

$$
-1<\left(\frac{C^{2}}{M G k}-1\right)\left(2+\frac{h k}{M G}\right)<0
$$

from where it follows

$$
-\frac{M G}{k}\left(1+\frac{C^{2}}{C^{2}-M G k}\right)<h<-\frac{2 G M}{k} .
$$

The semi-axis of the ellipse is determined by formulae

$$
a=\frac{p}{1-\varepsilon^{2}}, b=\frac{p}{\sqrt{1-\varepsilon^{2}}} .
$$


At

$$
h=-\frac{M G}{k}\left(1+\frac{C^{2}}{C^{2}-M G k}\right)
$$

the trajectory is a circle, at $h=-2 G M / k$ it is a parabola, and at $h>-2 G M / k$ it is a hyperbola. The constant of integration $h$, as usual, is determined from the initial condition: at $r=r_{0}, v=v_{0}$. According to (6)

$$
h=v_{0}^{2}-\frac{2 G M}{k} \mathrm{e}^{k / r_{0}},
$$

and condition (17) will be written in the form of

$$
v_{0}^{2}<\frac{2 G M}{r_{0}}\left(\frac{\mathrm{e}^{k / r_{0}}-1}{k / r_{0}}\right)=v_{*}^{2} .
$$

Therefore, at $v_{0}<v_{*}$ the trajectory is an ellipse, at $v_{0}=v_{*}$ it is a parabola, and at $v_{0}>v_{*}$ it is a hyperbola. $\lim _{k \rightarrow 0} v_{*}^{2}=2 G M / r_{0}=v_{*_{0}}^{2}, v_{* 0}=\sqrt{2 G M / r_{0}}$ is the second cosmic (escape) velocity by Newton theory, i.e. the initial velocity under which the body overcomes the gravitation of the body with mass $M$. And if $k>0$, according to (23) $v_{*}>v_{* 0}$, i.e. the second cosmic (escape) velocity under the interaction (1) is greater than the classic one which was to be expected.

Note, as well that the preservation in Maclaurin series of the functions $e^{k \mu}$ more components than in (10) brings to the calculation of the elliptic integrals and non-essential amendments to the parameters of trajectory.

\section{On Gravitational Radius of a Black Hole}

The body with mass $M$ will be dark (invisible or "Black Hole") if any body (particle) with mass $m$ and initial velocity, even equal to the speed of light $c$, cannot overcome the field of gravitation of the mass $M$. A natural question rises-what is the gravitational radius $R_{g}$ under the interaction (1). For the determination of the gravitational radius $R_{g}$ in Formula (23) the initial conditions will be: at $r_{0}=R_{g}, v_{*}=c$. We have

$$
\frac{2 G M}{R_{g}}\left(\frac{\mathrm{e}^{k / R_{g}}-1}{k / R_{g}}\right)=c^{2},
$$

Noting $\lim _{k \rightarrow 0} R_{g}=r_{g}$ and passing in (24) to the limit at $k \rightarrow 0$ we have

$$
\frac{2 G M}{r_{g}}=c^{2} \text { or } \frac{2 G M}{c^{2}}=r_{g}
$$

i.e. radius $r_{g}$ is the well-known gravitational radius under Newton classic central interaction.

Note $\gamma=k / R_{g}$, then from (24) taking into account (25) it follows

$$
R_{g}=r_{g} \frac{\mathrm{e}^{\gamma}-1}{\gamma}
$$

or 


$$
\frac{R_{g}}{r_{g}}=\frac{\mathrm{e}^{\gamma}-1}{\gamma}
$$

$\lim _{\gamma \rightarrow 0} R_{g} / r_{g}=1$ at $\gamma>0, R_{g}>r_{g}$ and from the graph of the function $R_{g} / r_{g}$ (see Figure 1) it follows that the gravitational radius $R_{g}$ under the interaction (1) compared with Newtonian gravitational radius $r_{g}$ can be arbitrarily large. From Formulae (24), (26) it follows that parameter $k$ is proportional to the gravitational radius $R_{g}\left(k=\gamma R_{g}\right)$. Setting the value $\gamma$ from graph (Figure 1 ) or by Formulae (27) $R_{g} / r_{g}$ will be determined, i.e. the gravitational radius $R_{g}$ itself. Then $k=\gamma R_{g}$.

In Table 1 for some values $\gamma$ the corresponding values $R_{g} / r_{g}$ are brought.

It is possible to do the opposite: to give the values $R_{g} / r_{g}$ and from equation (27) or Figure 1 determine $\gamma$ (Table 2), and hence the values $k\left(k=\gamma R_{g}\right)$.

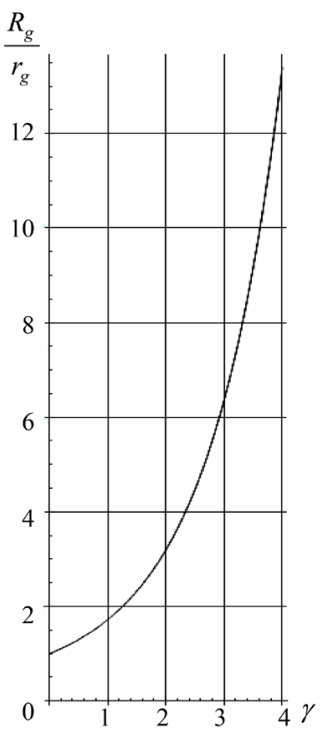

Figure 1. The dependence between $R_{g} / r_{g}$ and the intensity index of the gravitational field of a Black Hole $\gamma=k / R_{g}$.

Table 1. The values of $R_{g} / r_{g}$ corresponding to the given values of $\gamma$.

\begin{tabular}{cccccc}
\hline$\gamma$ & 1 & 2 & 3 & 5 & 10 \\
\hline$R_{g} / r_{g}$ & 1.718 & 3.194 & 6.362 & 29.483 & 2202.546 \\
& 50 & 100 & 200 & \multicolumn{1}{c}{1000} \\
& $1.037 \times 10^{20}$ & $2.689 \times 10^{41}$ & $3.61 \times 10^{84}$ & $1.97 \times 10^{431}$ \\
\hline
\end{tabular}

Table 2. The values of $\gamma$ corresponding to the given values of $R_{g} / r_{g}$.

\begin{tabular}{cccccc}
\hline$R_{g} / r_{g}$ & 1 & 2 & 3 & 5 & 10 \\
\hline$\gamma$ & 0 & 1.256 & 1.904 & 2.66 & 3.615 \\
& 50 & 100 & 200 & 500 & 1000 \\
& 5.647 & 6.475 & 7.285 & 8.335 & 9.118
\end{tabular}


The results obtained above permit us to draw a conclusion that Black Hole (Dark Body) may exist with arbitrarily large gravitational radius $R_{g}$; gravitation of the Black Hole (Dark Body) is not submitted to the classical (Newtonian) law of gravitation, but is submitted to the essentially powerful central gravitation (1). A lot of Black Hole (Dark Body) may exist. The last fact has long been confirmed by the astronomers [7]-[14].

All the Black Hole (Dark Body) will differ in power of the created by them gravitational field, i.e. by the value of the index $k$ of gravitational intensity. Using Formula (27) they can be renumbered according to the values increase of the index $k$.

Thus, Black Hole creates near to itself a stronger central force field of attraction than Newtonian, and submit the law

$$
\boldsymbol{F}=-G m M \frac{\mathrm{e}^{\gamma^{\frac{R_{g}}{r}}}}{r^{2}} \frac{\boldsymbol{r}}{|\boldsymbol{r}|}
$$

The Newtonian theory of gravitation is also capable of describing Black Hole, if the law of central attraction is taken in the form (28).

We also note that the "strong interaction", known in nuclear physics, is also exponential, but with a different potential compared with the potential corresponding to (28) [5].

\section{Discussion and Conclusions}

A new version of the central interaction of bodies is established:

$F=-G m M \mathrm{e}^{k / r} / r^{2}$ which at short distances describes more powerful, comparing with Newtonian one, gravitational interaction. Conditions, under which the movement trajectory is a conical section, are derived. It is shown that the Second cosmic (escape) velocity under the interaction (1) is much more than the Second cosmic (escape) velocity by Newton theory. The connection between the gravitational radius $R_{g}$ of the Black Hole and the gravitation intensity index " $k$ " of the gravitation center is found. It is shown that the gravitational radius of the Black Hole may be arbitrarily big. A lot of Black Holes may exist. They all differ in the value of the gravitation intensity index " $k$ ". The gravitation of the Black Hole (Dark Body) does not obey the classical (Newtonian) law of Gravitation, it obeys the law of the essentially powerful central gravitation (1) or (28).

\section{References}

[1] Kittel, Ch., Knight, W.D., et al. (1965) Mechanics (Berkeley Physics Course, v.1). Mc Graw-Hill, New York.

[2] Kaufman, W. (1977) The Cosmic Frontiers of General Relativity. Brown and Co, Boston, Toronto.

[3] Layzer, D. (1984) Constructing the Universe. Scientific American Books Inc., New York.

[4] Appell, P. (1960) Theoretical Mechanics. Fizmatlit, Moscow, 1.

[5] Wichman, E. (2010) Quantum Physics, (Berkeley Physics Course, v.4). Mc. Graw 
Hill, India.

[6] Aghalovyan, L.A. (2006) On Universal Central Strong (Weak) Interaction of Bodies and Particles. Reports of National Academy of Sciences of Armenia, 106, 238-244. (In Russian)

[7] Harms, R.J. and Holland, F.C., et al. (1994) HSTFOS Spectroscopy of M87: Evidence for a Disk of Ionized Gas around a Massive Black Hole. Astrophysical Journal, 435, 35-38. https://doi.org/10.1086/187588

[8] Kormendy, J. and Richstone, D. (1995) Inward Bound-The Search of Supermassive Black Holes in Galactic Nuclei. Annual Review of Astronomy and Astrophysics, 33, 581. https://doi.org/10.1146/annurev.aa.33.090195.003053

[9] Hehl, F.W. and Kiefer, C., et al. (1998) Black Hole: Theory and observation. Proceeding of the 179th W.E. Heraeus Seminar Held at Bad Honnef, Springer, Germany.

[10] Ellis, A. (1999) Black Holes.-Part 1-History. The Astronomical Society of Edinburgh Journal, 39.

[11] Genzel, R., Schodel, R., et al. (2003) The Stellar Cusp around the Supermassive Black Hole in the Galactic Center. Astrophysical Journal, 594, 812-832. https://doi.org/10.1086/377127

[12] Greighton, T. and Price, R. (2008) Supermassive Black Hole. Scholarpedia, 3, 1.

[13] McConnell, N., Ma, C., et al. (2011) Two Ten-Billion-Solar-Mass Black Holes at the Center of Giant Elliptical Galaxies. Nature, 480, 215-218. https://doi.org/10.1038/nature10636

[14] Mitra, A. (2012) Kruskal Coordinates and Mass of Schwarzschild Black Holes: No Finit Mass Black Hole at All. International Journal of Astronomy and Astrophysic, 2, 236-248. https://doi.org/10.4236/ijaa.2012.24031 